\title{
Radikalische Homo- und Copolymerisation von Styrol und Butylacrylat in überkritischem Kohlendioxid
}

\author{
Dissertation \\ zur Erlangung des Doktorgrades \\ der Mathematisch-Naturwissenschaftlichen Fakultäten \\ der Georg-August-Universität zu Göttingen
}

vorgelegt von

Almut Wahl

aus Kassel

Göttingen 1999 
D 7

Referent: $\quad$ Prof. Dr. M. Buback

Korreferent: Prof. Dr. K.-H. Hoyermann

Tag der mündlichen Prüfung: 26.01.2000 